\title{
Compulsive sexual behaviour in Parkinson's disease is associated with higher doses of levodopa
}

\section{Authors}

Pedro Barbosa ${ }^{1,2}$, Talyta Grippe ${ }^{3}$, Andrew J Lees ${ }^{1}$, Sean S O'Sullivan ${ }^{4}$, Atbin Djamshidian ${ }^{1,5^{*}}$, Thomas T Warner ${ }^{1,2^{*}}$

\section{Affiliation}

1. Reta Lila Weston Institute of Neurological Studies, UCL Institute of Neurology, London, UK

2. National Hospital for Neurology and Neurosurgery, Queen Square, London, UK

3. Department of Neurology, Faculty of Medicine, Centro Universitário de Brasília, Brasília, Brazil

4. Department of Neurology, Bon Secours Hospital, Cork, Ireland

5. Department of Neurology, Innsbruck Medical University, Innsbruck, Austria

\section{* Corresponding authors}

\section{Thomas T Warner}

Reta Lila Weston Institute of Neurological Studies, Institute of Neurology, University College London 1 Wakefield Street, WC1N1PJ, London, United Kingdom

Telephone: +4402076794246.

Email: t.warner@ucl.ac.uk

\section{Atbin Djamshidian}

Department of Neurology, Innsbruck Medical University, Austria

Anichstrasse 35; A-6020 Innsbruck; Austria

Tel +43/512/504/83197 Fax +43/512/504/23852

E-mail: atbin.djamshidian@gmail.com

Word count: 991

Key words: Compulsive sexual behaviour; Parkinson's disease; impulsive compulsive behaviours; levodopa. 


\section{INTRODUCTION}

Previous research estimates the lifetime prevalence of compulsive sexual behaviour (CSB) in individuals with PD to be $2.7 \%$. CSB has also been associated with male gender and earlier onset of PD. ${ }^{1}$ Although both dopamine agonists and to a lesser extent levodopa have been associated with impulsive compulsive behaviours (ICBs) ${ }^{2}$, it is still unclear whether higher levodopa doses are a risk factor for the development of CSB in PD patients.

\section{METHODS}

Patients with ICBs were identified from a database of individuals with PD and ICBs who were seen at the National Hospital for Neurology and Neurosurgery, Queen Square, London, UK and who had participated in 3 previous research projects over an eight-year period (from 2008 to 2016). Each project received approval from the local research ethics committee. All the ICB cases were recruited to research studies from PD clinics at the National Hospital and selected due to the reporting of ICBS. All cases underwent a thorough clinical investigation as well as a detailed semi-structured interview conducted by one of the authors. Hospital notes were reviewed by a movement disorder specialist (P.B.) for clinical and demographic data. Levodopa equivalent daily dose (LEDD) was calculated according to previously published guidelines. ${ }^{3}$ Data was analysed using the software SPSS@ 24.

\section{RESULTS}

In total, 128 patients with PD and ICBs were identified. Seventeen cases were excluded because data on dopaminergic treatment when the ICB was most active was incomplete. The remaining 111 patients were included in the analysis. Nearly $75 \%$ of the patients were males. The average age of PD onset for the entire cohort was 46.3 years, mean PD duration 11.3 years and mean age at ICBs 56.9 years. Dopamine agonists were used by $91 \%$ of the patients.

CSB was the most frequent ICB identified, present in $49.5 \%$ of the patients, followed by punding (43.2\%), compulsive shopping (38.7\%), pathological gambling (32.4\%), dopamine dysregulation syndrome (24.3\%) and compulsive eating (19.8\%). Multiple ICBs were present in 69 patients (62.1\%).

For statistical analysis we divided the cohort into two groups based on the presence of CSB: $\mathrm{CSB}^{+}(\mathrm{N}$ $=55)$ and $\operatorname{CSB}^{-}(N=56)$. The proportion of male individuals was higher in the $\operatorname{CSB}^{+}$group $(p<0.001)$ and these individuals developed ICBs at younger age $(p=0.02)$ (Table 1 ).

We did not find any differences between groups in the proportion of patients using levodopa, dopamine agonists, MAO inhibitors (MAOi), amantadine or COMT inhibitors (COMTi). Nine patients had not been exposed to dopamine agonists, 3 with CSB and 6 without. The $\operatorname{CSB}^{+}$group was using a higher dose of dopaminergic treatment and levodopa as measured by total LEDD ( $p=0.014)$, levodopa daily dose $(p=0.043)$, combined levodopa and COMTi LEDD $(p=0.039)$, and isolated COMTi LEDD $(p=0.026)$. DA LEDD $(p=0.802)$ and MAOi LEDD $(p=0.934)$ were similar between groups. Multiple ICBs were present in $48.2 \%$ of individuals without and $76.3 \%$ of individuals with CSB $(p=0.002)$ (table 1$)$. 
Table 1 - Clinical and demographic characteristics divided by groups

\begin{tabular}{|c|c|c|c|}
\hline & $\mathrm{CSB}^{+}$ & $\mathrm{CSB}^{-}$ & $\mathrm{p}$ \\
\hline$N=111$ & 55 & 56 & \\
\hline Proportion of males & 94.5 & 60.7 & $<0.001 *$ \\
\hline Mean age of PD onset in years & 44.6 & 48.0 & $0.079 * *$ \\
\hline Mean PD duration at onset of ICBs in years & 10.8 & 11.8 & $0.471 * *$ \\
\hline Mean PD duration at assessment in years & 11.8 & 13.2 & $0.344^{* *}$ \\
\hline Mean age at ICBs onset in years & 54.6 & 59.1 & $0.02 * *$ \\
\hline Multiple ICBs (\%) & 76.3 & 48.2 & $0.002 *$ \\
\hline Patients using levodopa (\%) & 98.1 & 91 & $0.206^{*}$ \\
\hline Patients using DA (\%) & 94.5 & 89.2 & $0.48^{*}$ \\
\hline Patients using MAOi (\%) & 36.3 & 26.7 & $0.312^{*}$ \\
\hline Patients using amantadine (\%) & 49 & 44.6 & $0.705^{*}$ \\
\hline Patients using COMTi (\%) & 49 & 53.5 & $0.706 *$ \\
\hline Levodopa daily dose (mg) & 994.5 & 704.9 & $0.043 * *$ \\
\hline DA LEDD (mg) & 385 & 357.8 & $0.802 * *$ \\
\hline MAOi LEDD (mg) & $97.5(N=20)$ & $96.67(\mathrm{~N}=15)$ & $0.934 * *$ \\
\hline Levodopa + MAOi LEDD (mg) & $911.5(\mathrm{~N}=20)$ & $696.7(N=15)$ & $0.114 * * *$ \\
\hline COMTi LEDD (mg) & $265.2(N=27)$ & $215.6(N=30)$ & $0.026 * *$ \\
\hline Levodopa + COMTi LEDD (mg) & $1068.5(\mathrm{~N}=27)$ & $874.5(N=30)$ & $0.039 * *$ \\
\hline Total LEDD (mg) & 1400.1 & 1163.6 & $0.014 * *$ \\
\hline Punding & 38.1 & 48.2 & $0.34^{*}$ \\
\hline Compulsive shopping & 43.6 & 33.9 & $0.333^{*}$ \\
\hline Pathological gambling & 30.9 & 33.9 & $0.840 *$ \\
\hline Dopamine dysregulation syndrome & 21.8 & 26.7 & $0.659 *$ \\
\hline Compulsive eating & 14.5 & 25 & $0.234^{*}$ \\
\hline
\end{tabular}

PD - Parkinson's disease; SD - standard deviation; ICBs - impulsive compulsive behaviours; DA- dopamine agonist; MAOi monoamine oxidase inhibitor; COMTi - Catechol-O-methyl transferase inhibitor; LEDD - levodopa equivalent daily dose.

*Chi-square test. ${ }^{* *}$ Mann-Whitney test. ${ }^{* * *}$ Independent samples t-test. Significant results in bold

The number of patients with DDS did not differ between the groups $(p=0.659)$, suggesting that the higher dose of levodopa in the $\mathrm{CSB}^{+}$group was not being driven by a higher proportion of dysregulators (Table 1).

\section{DISCUSSION}

CSB was the most frequently identified ICB in our cohort, affecting almost $50 \%$ of people with PDassociated ICBs. We report, for the first time, that PD patients with CSB tend to develop this abnormal behaviour at an earlier age and are more likely to develop multiple ICBs compared to PD patients with other ICBs.

Another novel finding is that patients with CSB were on higher doses of dopaminergic treatment as measured in LEDD. DA and MAOi dose, and the proportion of patients using DA, amantadine and MAOi did not differ between groups. However, patients with CSB were using a higher levodopa daily 
dose and higher COMTi doses than patients without CSB, although the proportion of patients using COMTi did not differ between groups. This suggests that higher dopaminergic stimulation, particularly higher doses of levodopa are a risk factor for the development of CSB. It is likely that higher doses of COMTi are not directly related to ICBs but are rather contributing to excessive dopaminergic stimulation by increasing the bioavailability of levodopa. This is corroborated by the fact that only half of the patients were using COMTi.

The association of abnormal sexual behaviour and levodopa was reported in the early days of levodopa use, years before dopamine agonists started being used for PD. Barbeau and colleagues treated 80 patients with Parkinson's disease with an average dose of $4.8 \mathrm{~g}$ of levodopa per day and reported that at least 4 males developed an increase in libido. ${ }^{4}$

Although the main risk factor for the development of impulse controls disorders in PD is the use of DAs ${ }^{2}$, levodopa has been found to be an important contributor to the development of ICBs in patients with PD receiving treatment with DAs. ${ }^{5}$ Interestingly, despite finding that patients with CSB were on higher doses of levodopa, the proportion of other types of ICBs was similar between groups, indicating that even though higher levodopa doses are associated with CSB and DDS, these abnormal behaviours are not more likely to occur together.

By including only patients that participated in previous research projects, it is possible that we have missed patients with ICBs that were diagnosed during a regular outpatient's appointment. However, since we were interested in assessing the prevalence of CSB among patients with established ICBs we believe this approach minimised the possibility of including false positives.

\section{CONCLUSION}

The data from this study suggest that CSB is more frequent in males and tends to appear earlier than other ICBs. CSB may be the most frequent ICB associated with PD. Furthermore, patients with CSB are more likely to develop multiple ICBs. When compared to other types of ICBs, this behavioural addiction appears to be driven by higher levodopa doses. Data from larger studies are needed to confirm these novel findings.

\section{Acknowledgements}

We would like to thank the Reta Lila Weston Institute of Neurological Studies for the support received during this study.

Pedro Barbosa is supported by a grant from the Brazilian National Council for Scientific and Technologic development (Conselho Nacional de Desenvolvimento Cientifico e Tecnologico, CNPQ).

Contributors Study concept and design: PB, AJL, SOS, AD, TTW. Gathering of data: PB, TG, SOS, AD. Analysis and interpretation of data: PB, TG, AJL, SOS, AD, TTW. Drafting of the manuscript: PB. Critical revision of the manuscript: TG, AJL, SOS, AD, TTW. 


\section{Competing Interests}

Pedro Barbosa received support to attend academic meetings from Britannia Pharmaceuticals and the Movement Disorders Society, and has received a grant from Britannia Pharmaceuticals.

Talyta Grippe has nothing to disclose.

Andrew Lees is funded by the Reta Lila Weston Institute of Neurological Studies, University College London, Institute of Neurology and reports consultancies for: Britannia Pharmaceuticals and BIAL Portela. He also reports grants and/or research support: from the Frances and Renee Hock Fund, and honoraria from Britannia, Profile Pharma, UCB, Roche, Lundbeck, Teva, BIAL, Nordiclnfu Care, NeuroDerm.

Sean O'Sullivan has received support to attend academic meetings and honorarium from Teva, Lundbeck pharmaceuticals, Eisai, UCB Pharma, AbbVie Pharma

Atbin Djamshidian has nothing to disclose.

Tom Warner has received support to attend academic meetings from Britannia Pharmaceuticals

\section{REFERENCES}

1. Nakum S, Cavanna AE. The prevalence and clinical characteristics of hypersexuality in patients with Parkinson's disease following dopaminergic therapy: A systematic literature review. Parkinsonism \& related disorders 2016;25:10-6. doi: 10.1016/j.parkreldis.2016.02.017

2. Weintraub D, Koester J, Potenza MN, et al. Impulse control disorders in Parkinson disease: a crosssectional study of 3090 patients. Archives of neurology 2010;67(5):589-95. doi: 10.1001/archneurol.2010.65

3. Tomlinson CL, Stowe R, Patel S, et al. Systematic review of levodopa dose equivalency reporting in Parkinson's disease. Movement disorders : official journal of the Movement Disorder Society 2010;25(15):2649-53. doi: 10.1002/mds.23429

4. Barbeau A. L-dopa therapy in Parkinson's disease: a critical review of nine years' experience. Canadian Medical Association journal 1969;101(13):59-68. [published Online First: 1969/12/27]

5. Hassan A, Bower JH, Kumar N, et al. Dopamine agonist-triggered pathological behaviors: surveillance in the PD clinic reveals high frequencies. Parkinsonism \& related disorders 2011;17(4):260-4. doi: 10.1016/j.parkreldis.2011.01.009 